\title{
Clusterin expression in cutaneous CD30-positive lymphoproliferative disorders and their histologic simulants
}

Background: Clusterin is a ubiquitous $80 \mathrm{kDa}$ heterodimeric glycoprotein previously shown to be expressed on tumor cells of systemic and, to a lesser extent, primary cutaneous anaplastic large cell lymphoma (PC-ALCL). Lymphomatoid papulosis (LyP), an important differential diagnosis of ALCL, has been studied for clusterin expression in only a small number of cases. The aim of this study was to compare clusterin immunostaining patterns in $\mathrm{LyP}$ and other cutaneous histologic simulants with those of PC-ALCL.

Methods: Formalin-fixed, paraffin-embedded sections of PC-ALCL (6), LyP (20), mycosis fungoides with large cell transformation (MF-LCT, 12), pityriasis lichenoides et varioliformis acuta (PLEVA, 12), arthropod bite reaction (ABR, 12) and lymphomatoid reactions (LR, 9) were immunostained for clusterin and evaluated for staining pattern and distribution. All diagnoses were made with clinicopathologic correlation.

Results: Characteristic dot-like Golgi staining was identified in 10/ 20 LyP (50\%), 4/6 PC-ALCL (67\%) and 9/12 MF-LCT (75\%). Two of 12 PLEVA (17\%), 1 of 12 ABR (8\%) and 2 of 8 LR (25\%) had lymphocytes $(<25 \%)$ with diffuse cytoplasmic staining. Dermal dendritic cells stained strongly for clusterin. High background staining occurred in some cases.

Conclusion: Glusterin immunostaining does not reliably distinguish between LyP, PG-ALCL or MF-LGT, but could distinguish LyP from its reactive histologic simulants.

Olsen SH, Ma L, Schnitzer B, Fullen DR. Clusterin expression in cutaneous CD30-positive lymphoproliferative disorders and their histologic simulants.

J Cutan Pathol 2009; 36: 302-307. (c) Blackwell Munksgaard 2008.

\section{Stephen H. Olsen ${ }^{1}$, Linglei $\mathrm{Ma}^{1,2}$, Bertram Schnitzer ${ }^{1}$ and Douglas R. Fullen ${ }^{1,2}$}

${ }^{1}$ Department of Pathology and ${ }^{2}$ Department of Dermatology, University of Michigan Medical Center, Ann Arbor, MI, USA
Douglas R. Fullen, MD, M3261, Medical Science I, Department of Pathology, University of Michigan Medical Center, 1301 Catherine, Ann Arbor, MI 48109-0602, USA

Tel: +17347644460

Fax: +17347644690

e-mail: dfullen@umich.edu

Accepted for publication March 17, 2008
Clusterin is a ubiquitous $80 \mathrm{kDa}$ heterodimeric glycoprotein found in many tissues and in virtually all body fluids, including plasma, urine, seminal fluid and breast milk. The clusterin gene is located on human chromosome 8 and is highly conserved across mammalian species. ${ }^{1,2}$ This protein is thought to play a role in reproduction, lipid transport, apoptosis, cell survival and complement regulation; moreover, it has been shown to be upregulated in areas of tissue remodeling, in apoptotic disease states and in response to injury. ${ }^{1}$
Wellman et al. identified clusterin as a specific marker of systemic anaplastic large cell lymphoma (S-ALCL) through hybridization of complementary DNA to gene expression arrays followed by confirmatory Western blot and immunohistochemical studies. The authors proposed that the expression of clusterin could be used diagnostically to distinguish S-ALCL, the cells of which were positive in all 36 cases evaluated, from primary cutaneous ALCL (PGALCL), whose cells were negative in all nine cases studied. Obviously, this result has clinical importance 
Clusterin and CD30+ lymphoproliferative disorders

because of differences in treatment and prognosis between the two processes. ${ }^{3}$ Subsequent studies, however, have shown that clusterin is expressed in both S-ALGL and PC-ALCL, although to a lesser extent in the latter. ${ }^{4,5}$

Clusterin expression in lymphomatoid papulosis $(\mathrm{Ly} \mathrm{P})$, an important differential diagnosis of ALCL in the skin, has been reported in only a small number of cases in the literature. Saffer et al. observed positive clusterin staining in only one of seven cases of $\mathrm{LyP}^{4}$ Similarly, clusterin expression in large cells of mycosis fungoides with large cell transformation (MF-LCT), another histologic mimic of ALCL, has been evaluated in only a few cases in the literature. ${ }^{4,6}$ To our knowledge, clusterin expression has not been described in other cutaneous lesions that resemble LyP clinically and histologically, such as lymphomatoid reactions (LR) to drugs or other stimuli, arthropod bite reactions (ABR) and pityriasis lichenoides et varioliformis acuta (PLEVA). The goal of this study was to evaluate and compare clusterin immunostaining patterns in $\mathrm{Ly} \mathrm{P}$ and the aforementioned cutaneous lymphoid lesions with those of PGALCL.

\section{Materials and methods}

Following approval by the University of Michigan Institutional Review Board, paraffin-embedded tissue blocks and slides from 80 skin biopsies with histologic diagnoses of LyP, ALCL, MF-LCT, ABR, PLEVA and LR were retrieved from the surgical pathology archives. Following clinicopathologic review, the 80 cases were classified as 6 ALCL, 20 LyP, 12 PLEVA, 12 ABR, 12 MF-LCT and 9 LR. Three of the six ALCL cases were of PC type. The LR occurred in response to a variety of antigenic stimuli, including drugs (minocycline, ampicillin, intravenous immunoglobulin, chemotherapy for myeloid leukemia), ${ }^{7}$ tattoo pigment and treponemal infection. A specific etiology was not established in three cases. Nine cases were excluded from analysis because of either lack of sufficient tissue (four cases) or change in diagnosis after clinicopathologic review (five cases), resulting in 71 cases for evaluation.

Formalin-fixed, paraffin-embedded tissue sections were immunostained for clusterin. Briefly, deparaffinized and rehydrated tissue sections, $4-\mu \mathrm{m}$ thick, were stained with a primary monoclonal antibody to clusterin (1 : 200; Upstate Cell Signalling Solutions, Lake Placid, NY, USA) following antigen retrieval with $1 \mathrm{mmol} / \mathrm{l}$ ethylene-diamine-tetrasacetia acid (EDTA) buffer ( $\mathrm{pH} \mathrm{8.0)} \mathrm{in} 95^{\circ} \mathrm{C}$ water bath for $20 \mathrm{~min}$. Immunohistochemical staining was performed on a DAKO Autostainer (DAKO, Carpinteria, CA, USA) using DAKO LSAB + and a 3,3'-diaminobenzidine chromogen.
Immunostained sections from each case were examined for a characteristic dot-like Golgi staining pattern, as well as membranous or diffuse cytoplasmic staining patterns, by cells within the infiltrate, especially the large atypical lymphoid cells. The degree of positivity was recorded semiquantitatively as percentage of infiltrating cells $(<1 \%, 1-25 \%, 25-$ $50 \%$ and $>50 \%$ ) by each of the authors, including two board-certified dermatopathologists (L. M. and D. F.).

\section{Results}

The data for clusterin are shown in Table 1. Characteristic dot-like perinuclear or Golgi staining was identified in 4/6 PG-ALCL (67\%), 10/20 LyP $(50 \%)$ and $9 / 12$ MF-LCT $(75 \%)$. While 2/12 PLEVA (17\%), 1/12 ABR (8\%) and 2/8 LR (25\%) had clusterin staining in a small percentage of cells $(<25 \%)$, the staining pattern was diffuse and cytoplasmic without Golgi accentuation, which differed from LyP, PG-ALCL and MF-LCT. With respect to the LyP cases, 8 of 15 type A lesions, 0 of 1 type $B$ lesion and 2 of 4 type $\mathrm{C}$ lesions showed clusterin expression. The majority of cases contained only small percentages $(<25 \%)$ of cells staining positive for clusterin. However, higher percentages of positive cells were noted in two cases of ALCL $(25-50 \%, 1$ and $50-75 \%, 1)$, two cases of LyP (25-50\%) and four cases of MF-LCT (25-50\%, 3 and 50-75\%, 1) (Fig. 1).

A subset of dermal dendritic cells within the lymphoid infiltrates showed intense and diffuse cytoplasmic staining for clusterin in many cases (Fig. 2). While these dendritic cells with clusterin expression were observed in each of the diagnoses included in this study, they were more prominent in MF-LCT (11/12) and LR (7/9) compared with LyP (8/20), ALCL (2/6) and ABR (6/12). In a parallel study, we evaluated 12 cases of Langerhans cell histiocytosis (LCH) and found that 3 cases $(25 \%)$ showed focal and weak cytoplasmic staining for clusterin (data not shown).

Table 1. Data on clusterin expression in CD30-positive Iymphoproliferative disorders and histologic simulants

\begin{tabular}{lcccll}
\hline \multirow{5}{*}{ Diagnosis } & Cases $(\mathrm{n})$ & $<1$ & $1-25 \%$ & $25-50 \%$ & $50-75 \%$ \\
\cline { 3 - 6 } & 6 & 2 & 2 & 1 & 1 \\
ALCL & 20 & 10 & 8 & 2 & 0 \\
LyP & 12 & 3 & 5 & 3 & 1 \\
MF-LCT & 12 & 10 & 2 & 0 & 0 \\
PLEVA & 12 & 11 & 1 & 0 & 0 \\
ABR & 9 & 6 & 3 & 0 & 0 \\
LR & 71 & 42 & 21 & 6 & 2 \\
Total & & &
\end{tabular}

$A B R$, arthropod bite reaction; $A L C L$, anaplastic large cell lymphoma; $L R$, lymphomatoid reactions; LyP, Iymphomatoid papulosis; MF-LCT, mycosis fungoides with large cell transformation; PLEVA, pityriasis lichenoides et varioliformis acuta. 


\section{Olsen et al.}
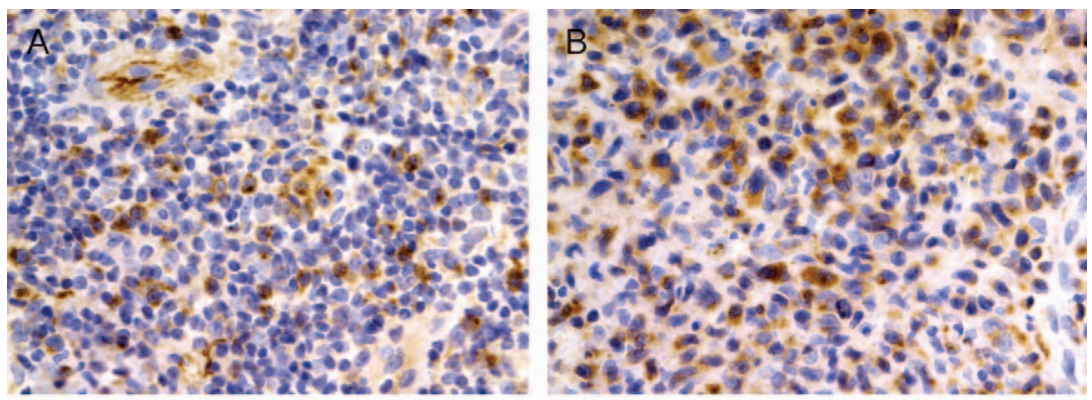

Fig. 1. Clusterin immunostaining with a Golgi pattern in various CD30-positive lymphoproliferative disorders and reactive conditions: (A) lymphomatoid papulosis with $<25 \%$ of lesional cells staining. B) Primary cutaneous anaplastic large cell lymphoma (PC-ALCL) with $25-50 \%$ of lesional cells positive for clusterin. C) PC-ALCL with 50 $75 \%$ of lesional cells expressing clusterin. D) Lymphomatoid reaction with a low percentage of clusterin-positive cells with diffuse cytoplasmic staining (arrowheads); note also the presence of clusterin-positive dendritic cells and non-specific background staining.

High background staining for clusterin occurred in a significant number of cases and was especially prominent in areas of ulceration, hemorrhage or tissue necrosis (Fig. 3). In some cases, the extent of background staining hindered the evaluation of the staining patterns within the lymphoid infiltrates.

\section{Discussion}

LyP and PG-ALCL comprise a spectrum of CD30positive lymphoproliferative disorders of the skin. These disorders are considered low-grade malignancies characterized by lesions that have a tendency to spontaneously regress and a relatively indolent clinical course with an overall favorable prognosis. ${ }^{8-10}$ These lymphoproliferative processes may share histologic and immunophenotypic features, making their

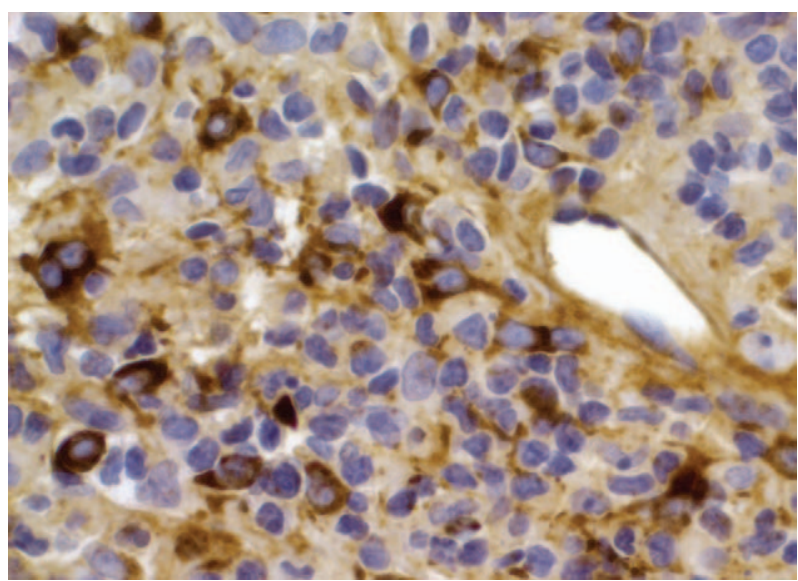

Fig. 2. Clusterin immunostaining of dendritic cells is seen in a lymphomatoid drug reaction. distinction problematic. ${ }^{9,11}$ LyP and ALCL have variable numbers of large CD30-positive lymphoid cells with anaplastic cytomorphology. ${ }^{9,12}$ The type $\mathrm{C}$ variant of $\mathrm{LyP}$ has a predominance of $\mathrm{CD} 30$-positive cells and may be histologically indistinguishable from PC-ALCL. This pattern can also be seen in S-ALCL secondarily involving the skin and in MF-LCT. Both disorders are important to distinguish from LyP and PG-ALCL because of the more aggressive clinical course in the former two entities. ${ }^{13-15}$ Type A (histiocytic) LyP contains similar CD30-positive tumor cells, but these large cells are admixed with

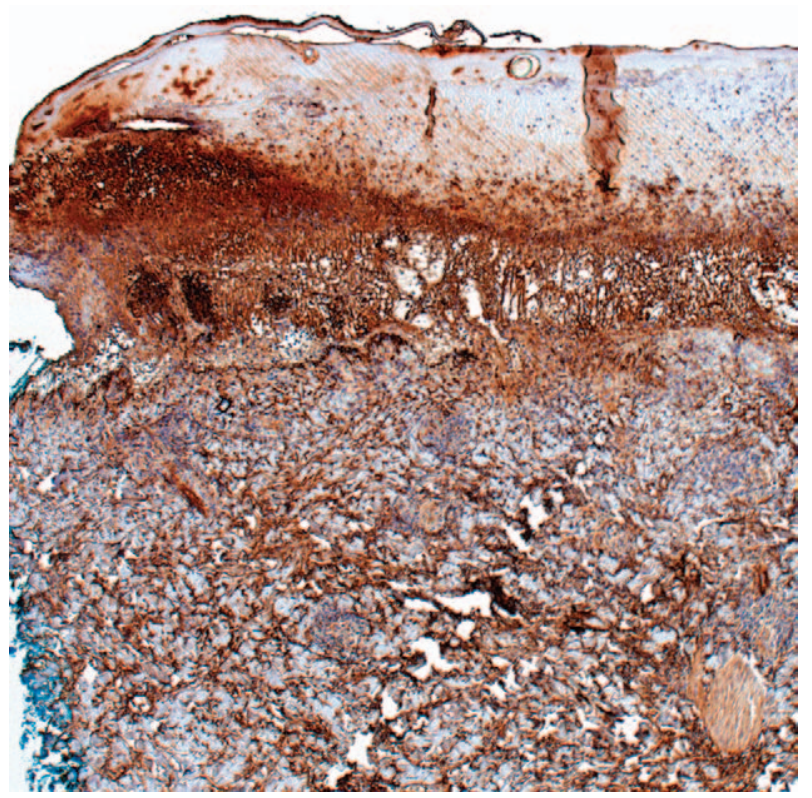

Fig. 3. Background staining of clusterin is present in association with epidermal necrosis and ulceration secondary to an arthropod bite reaction. 
Clusterin and CD30+ lymphoproliferative disorders

other inflammatory cells like neutrophils, eosinophils and histiocytes in the dermis. ${ }^{9,11}$ Thus, the differential diagnosis of type A LyP includes a variety of reactive conditions in the skin, namely PLEVA, ABR and some LR. ${ }^{16}$ Similar to type A LyP, these reactive processes may also contain some CD30-positive large lymphoid cells that intermingle with other inflammatory cells. ${ }^{7,9,17}$ Thus, it would be useful to identify an immunohistochemical marker that could distinguish ALCL from LyP or LyP from its histologic mimickers.

Wellman et al. initially identified clusterin in cases of S-ALCL but not in any cases of PG-ALCL. ${ }^{3}$ Subsequent studies refuted this finding by showing clusterin staining in both types of ALCL, although the data were somewhat variable. ${ }^{4-6}$ In two of these studies, fewer PG-ALCL lesions (41-56\%) were positive compared with S-ALCL secondarily involving the skin $(75 \%)$ and S-ALCL occurring at extracutaneous sites (82-95\%). ${ }^{4,5}$ One study reported clusterin expression in all cases of ALCL regardless of whether the process was cutaneous or systemic. ${ }^{6}$ A perinuclear dot-like Golgi staining pattern, with or without diffuse cytoplasmic or membranous pattern, within the cytoplasm of large atypical lymphoid cells had previously been reported to be characteristic of ALCL. ${ }^{3,6}$ However, some of these studies have also included cells with diffuse cytoplasmic or membranous staining but without Golgi staining as positive in their results. In the prior studies, clusterin staining patterns or proportions of positive cells were not described in (1/7) LyP or (1/2) MF-LCT cases., ${ }^{4} 6$ Clusterin expression has not been studied in the reactive cutaneous processes that may simulate $\mathrm{LyP}$ clinically and histologically and that may contain CD30-positive large lymphoid cells.

The presence of clusterin expression in $67 \%$ of cutaneous ALCL in our study and the presence of low percentages of positive cells within the infiltrate $(<25 \%$ in most cases $)$ are consistent with previous published data. ${ }^{4,5}$ In contrast to Nascimento et al., who reported clusterin staining in all of their cutaneous ALCL cases, the other previous studies found lower positivity in number of cases and proportions of positive cells in cutaneous relative to nodal or secondary cutaneous ALCL. ${ }^{4-6}$ The reason for the disparity in staining results between the different studies is not entirely clear but probably is multifactorial. The staining results may have been influenced by the immunohistochemical method used. A few of the previous studies used similar heat-induced antigen retrieval in citrate buffer at $\mathrm{pH}$ 6.0 prior to clusterin staining. Like Lae et al., we found in preliminary results that pretreatment with EDTA $\mathrm{pH} 8.0$ resulted in a crisper staining pattern and less background staining when compared with the citrate buffer method. Nevertheless, we noted high background staining in a subset of our cases, particularly when ulceration, hemorrhage or tissue necrosis was prominent. This finding can be explained by the presence of clusterin in normal serum and its extravasation into areas of tissue injury. Moreover, it is possible that clusterin expression is upregulated in lymphoid cells and/or dermal dendritic cells and/or excreted extracellularly in response to inflammatory mediators at the site of tissue injury. Regardless of the cause, this non-specific background staining can certainly hinder the interpretation of specific staining. We speculate that the stromal collagen and/or dendritic cells of the dermal milieu could downregulate clusterin expression in lymphoid cells when compared with extracutaneous lesions, although this hypothesis would not explain the findings by Nascimento et al.

Contrary to the previous study that evaluated clusterin expression in LyP, we found a substantially higher percentage of positive cases $(50 \%)$, similar to our results for ALCL. With the exception of two LyP cases, which had up to $50 \%$ of lesional cells staining for clusterin, the remainder of the positive cases of $\mathrm{Ly} P$ had a low percentage $(<25 \%)$ of clusterin-positive cells. Fifteen of our LyP cases were the most common type (type A), whereas four were type C (ALCL like) and one was type $\mathrm{B}$ (MF like). Ten of our LyP cases were positive for clusterin, including 8 of $15(53 \%)$ type A lesions and 2 of $4(50 \%)$ type C lesions; only one type B lesion was included in our study and was negative. The difference between our staining results in LyP and those of Saffer et al. could be explained by the difference in the type of LyP cases included, variability in tissue fixation of cases and the number of cases evaluated. ${ }^{4}$ Our study included all three types of LyP lesions, whereas the types of LyP lesions studied by Saffer et al. were not specified. We evaluated cases that were obtained within our institution, as opposed to Saffer et al. who stated that their cases included internal and referral cases that may have had significant differences in tissue fixation.

Previous data on MF-LCT are difficult to interpret because of the exceedingly low number of cases examined for clusterin expression. ${ }^{4,6}$ The majority of cases $(75 \%)$ in our study were positive and most showed a low percentage of clusterin-positive cells; however, four cases of MF-LCT had between 25\% and $75 \%$ of clusterin-positive cells. Nascimento et al. found the dot-like Golgi pattern to be specific for either primary or secondary ALCL. This Golgi pattern, however, was very prominent in our cases of MF-LCT. These findings highlight the non-specific nature of clusterin expression and, because of the differences in prognosis of these lesions, raise doubts about its clinical utility.

Clusterin expression was also found in a small subset of reactive conditions that included PLEVA, $\mathrm{ABR}$ and $\mathrm{LR}$ to various antigenic stimuli, such as 


\section{Olsen et al.}

drugs, tattoo pigment and infectious agents. A smaller percentage of these lesions were positive for clusterin when compared with LyP lesions, only a low percentage $(<25 \%)$ of lesional cells were positive in each category, and the staining pattern of positive cells in the infiltrate was diffuse and cytoplasmic, which differed from the perinuclear dot-like Golgi pattern of staining in LyP. Thus, this disparity in staining pattern was useful in distinguishing these reactive cutaneous infiltrates from LyP.

Intense, diffuse cytoplasmic staining of clusterin has been consistently noted in follicular dendritic cells of lymph nodes and fibroblastic reticular cells in lymph nodes and bone marrow. ${ }^{3-6}$ In fact, clusterin has been shown to be a highly specific marker for follicular dendritic cell sarcoma. ${ }^{18}$ However, the authors in that study did not specifically evaluate clusterin expression in dermal dendritic cells, which we found to have a dense and diffuse cytoplasmic staining pattern similar to that described for follicular dendritic and fibroblastic reticular cells. These clusterin-positive dendritic cells were most prominent in LR and, curiously, in MF-LCT. In a parallel study of 12 cases of LCH involving the skin, Langerhans cells lacked the strong diffuse clusterin positivity that was seen in the dendritic cells of some of our cases, with only $3(25 \%)$ cases showing weak and focal positive staining. In a study comparing follicular dendritic cell tumor to other dendritic cell tumors, Grogg et al. reported a higher percentage $(8 / 14,57 \%)$ of $\mathrm{LCH}$ lesions positive for clusterin, some with a moderate degree of staining. ${ }^{19}$ However, the authors do not specify the anatomic location of their LCH lesions. Our results suggest that the clusterin-positive dermal dendritic cells in our cases are probably not Langerhans cells. However, we did not perform additional studies, such as double-labeling immunohistochemistry, in an attempt to further delineate the immunophenotype of these clusterin-positive dendritic cells.

In summary, clusterin expression is observed in the large atypical lymphoid cells occurring in the spectrum of CD30-positive lymphoproliferative disorders, as well as in MF-LCT, involving the skin and cannot be used reliably to distinguish among these entities. Clusterin also is expressed on a small subset of inflammatory cells within the infiltrate of some cases of PLEVA, ABR and LR, albeit in a lower percentage of cases and in a diffuse cytoplasmic pattern rather than the Golgi pattern seen in the large lymphoid cells of LyP. This difference in staining pattern could discriminate between these reactive processes and type A LyP, although only a small number and limited types of LR were included. We did not attempt to distinguish between S-ALCL and PG-ALCL because this has been done in prior studies. Clusterin exhibits high background staining, particularly in areas of ulceration, necrosis and hemorrhage, in some cases, which limits its diagnostic usefulness in dermatopathology. Clusterin also intensely labels a subset of dermal dendritic cells within malignant and reactive lymphoid infiltrates of the skin in some cases. Further studies may shed light on the role of clusterin in the dermal dendritic microenvironment.

\section{Acknowledgement}

This work was presented at the 43rd Annual Meeting of the American Society of Dermatopathology in Chicago, IL, USA, in October 2006.

\section{References}

1. Jones SE, Jomary C. Clusterin. Int J Biochem Cell Biol 2002; 34: 427.

2. Rosenberg ME, Silkensen J. Clusterin: physiologic and pathophysiologic considerations. Int J Biochem Cell Biol 1995; 27: 633.

3. Wellman A, Thieblemont C, Pittaluga S, et al. Detection of differently expressed genes in lymphomas using cDNA arrays: identification of clusterin as a new diagnostic marker for anaplastic large cell-lymphomas. Blood 2000; 96: 398.

4. Saffer H, Wahed A, Rassidakis GZ, Medeiros LJ. Clusterin expression in malignant lymphomas: a survey of 266 cases. Mod Pathol 2002; 15: 1221.

5. Lae ME, Ahmed I, Macon WR. Clusterin is widely expressed in systemic anaplastic large cell lymphoma but fails to differentiate primary from secondary cutaneous anaplastic large cell lymphoma. Am J Clin Pathol 2002; 118: 773.

6. Nascimento AF, Pinkus JL, Pinkus GS. Clusterin, a marker for anaplastic large cell lymphoma immunohistochemical profile in hematopoietic and nonhematopoietic malignant neoplasms. Am J Clin Pathol 2004; 121: 709.

7. Su LD, Duncan LM. Lymphoma and leukemia-associated cutaneous atypical CD30 + T-cell reactions. J Cutan Pathol 2000; 27: 249 .

8. Willemze R, Meijer CJ. Primary cutaneous CD30-positive lymphoproliferative disorders. Hematol Oncol Clin N Am 2003; 17: 1319.

9. Kempf W. CD30+ lymphoproliferative disorders: histopathology, differential diagnosis, new variants, and simulants. J Cutan Pathol 2006; 33 (Suppl. 1): 58.

10. Kadin ME. Pathobiology of CD30+ cutaneous T-cell lymphomas. J Cutan Pathol 2006; 33 (Suppl. 1): 10.

11. El Shabrawi-Caelen L, Kerl H, Cerroni L. Lymphomatoid papulosis: reappraisal of clinicopathologic presentation and classification into subtypes A, B, and C. Arch Dermatol 2004; 140: 441.

12. Kadin ME, Carpenter C. Systemic and primary cutaneous anaplastic large cell lymphomas. Semin Hematol 2003; 40: 244.

13. Willemze R, Jaffe ES, Burg G, et al. WHO-EORTC classification for cutaneous lymphomas. Blood 2005; 105: 3768.

14. Vergier B, deMuret A, Beylot-Barry M, et al. Transformation of mycosis fungoides: clinicopathologic and prognostic features from 45 cases. Blood 2000; 95: 2212.

15. Diamandidou E, Colome-Grimmer M, Fayad L, Duvic M, Kurzrock R. Transformation of mycosis fungoides/Sezary syndrome: clinical characteristics and prognosis. Blood 1998; 92: 1150. 


\section{Clusterin and CD30+ lymphoproliferative disorders}

16. Gilliam AG, Wood GS. Cutaneous lymphoid hyperplasias. Semin Cutan Med Surg 2000; 19: 133.

17. Cepeda LT, Pieretti M, Chapman SF, Horenstein MG. CD30positive atypical lymphoid cells in common non-neoplastic cutaneous infiltrates rich in neutrophils and eosinophils. Am J Surg Pathol 2003; 27: 912.

18. Grogg KL, Macon WR, Kurtin PJ, Nascimento AG. A survey of clusterin and fascin expression in sarcomas and spindle cell neoplasms: strong clusterin immunostaining is highly specific for follicular dendritic cell tumor. Mod Pathol 2005; 18: 260.

19. Grogg KL, Lae ME, Kurtin PJ, Macon WR. Clusterin expression distinguishes follicular dendritic cell tumors from other dendritic cell neoplasms: report of a novel follicular dendritic cell marker and clinicopathologic data on 12 additional follicular dendritic cell tumors and 6 additional interdigitating dendritic cell tumors. Am J Surg Pathol 2004; 28: 988. 